\title{
Reproducibility, Preservation, and Access to Research with ReproZip and ReproServer
}

Vicky Steeves ${ }^{1,2}$ | Librarian for Research Data Management \& Reproducibility Remi Rampin ${ }^{2}$ | Research Software Engineer

${ }^{1}$ Division of Libraries, ${ }^{2}$ Center for Data Science | New York University Slides: osf.io/4uc3p 


\section{Reproducibility....}

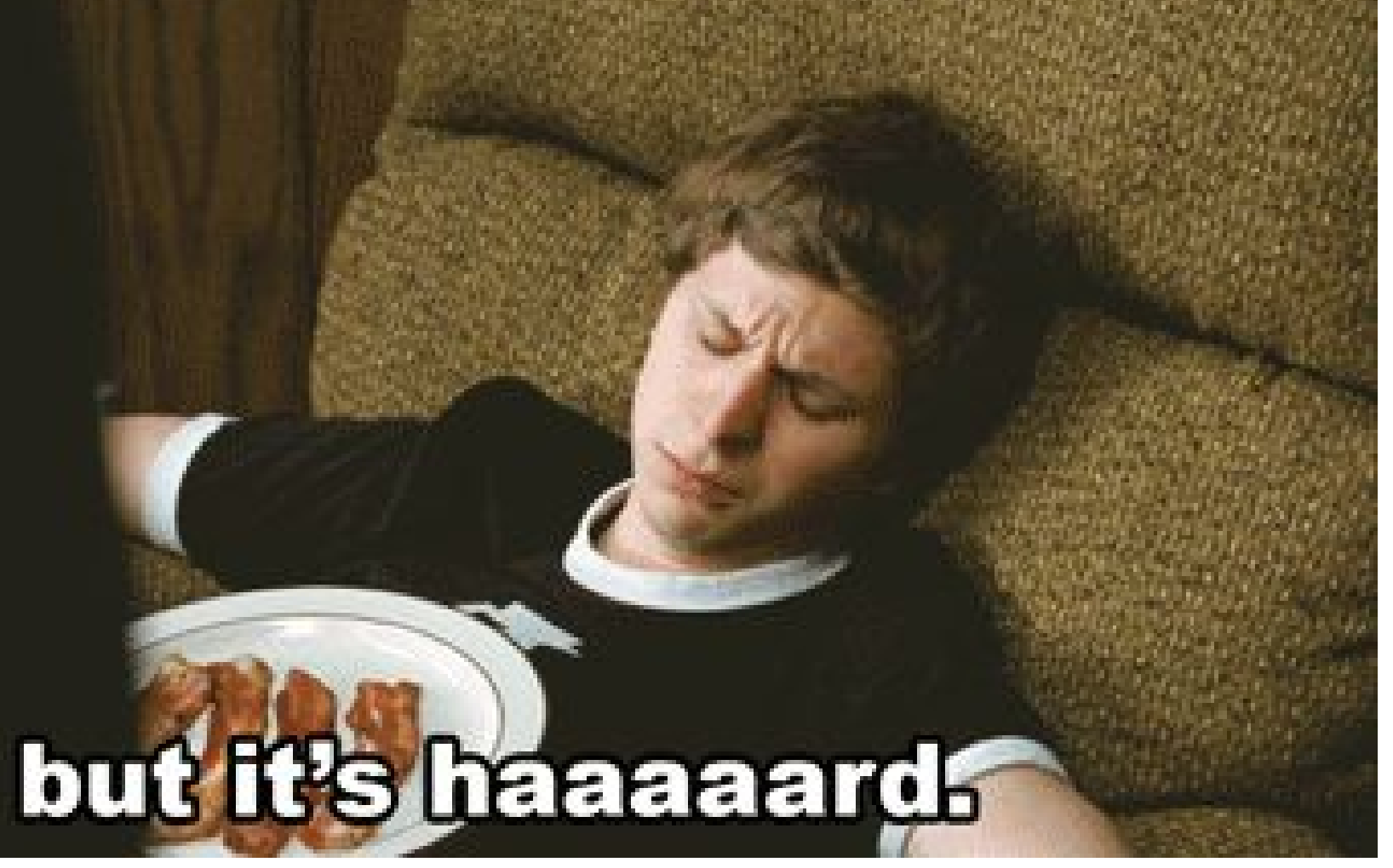




\section{As all things, reproducibility is defined as a spectrum}

Reviewable Research: Sufficient detail for peer review \& assessment.

Replicable Research: Tools are available to duplicate the author's results using their data. Confirmable Research: Main conclusions can be attained independently without author's software.

Auditable Research: Process \& tools archived such that it can be defended later if necessary. Open/Reproducible Research: Auditable

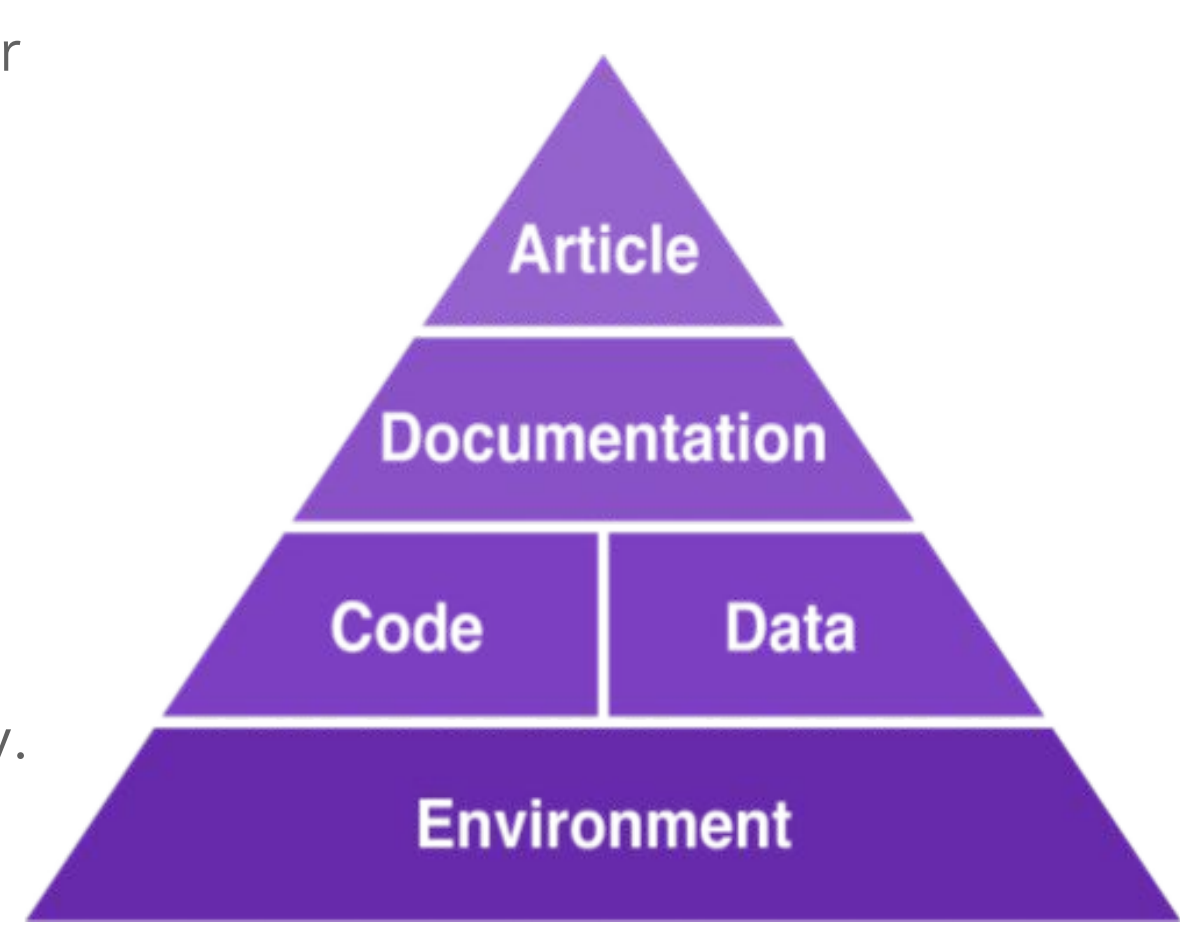
research made openly available 


\section{ReproZip tries to solve...}

\section{Workload \& Time Challenges}

It is a time commitment to get data and code ready to share, and to share it

Otherwise known as...

\section{the Incentive Problem}

Reproducibility takes time, and is not always valued by the academic reward structure

"Insufficient time is the main reason why scientists do not make their data and experiment available and reproducible." Carol Tenopir, Beyond the PDF2 Conference
" $77 \%$ claim that they do not have time to document and clean up the code." Victoria Stodden, Survey of the Machine Learning Community - NIPS 2010 


\section{ReproZip tries to solve...}

\section{Technical Obsolescence}

Technology changes affect the reproducibility

\section{Normative Dissonance ${ }^{1}$}

Espoused values don't always match practice

Otherwise known as...

\section{The Pipeline Problem}

Reproducibility requires skills that are often not included in most curriculums!

"It would require huge amount of effort to make our code work with the latest versions of these tools." Collberg et al., Repeatability and Benefaction in Computer Systems Research, University of Arizona TR 14-04 


\section{Even if runnable, results may differ...}

The Effects of FreeSurfer Version, Workstation Type, and Macintosh Operating System Version on Anatomical Volume and Cortical Thickness Measurements

We investigated the effects of data processing variables such as FreeSurfer version (v4.3.1, v4.5.0, and v5.0.0), workstation (Macintosh and Hewlett-Packard), and Macintosh operating system version (OSX 10.5 and OSX 10.6). Significant differences were revealed between FreeSurfer version v5.0.0 and the two earlier versions. [...] About a factor two smaller differences were detected between Macintosh and Hewlett-Packard workstations and between OSX 10.5 and OSX 10.6 


\section{The main problem ReproZip solves}

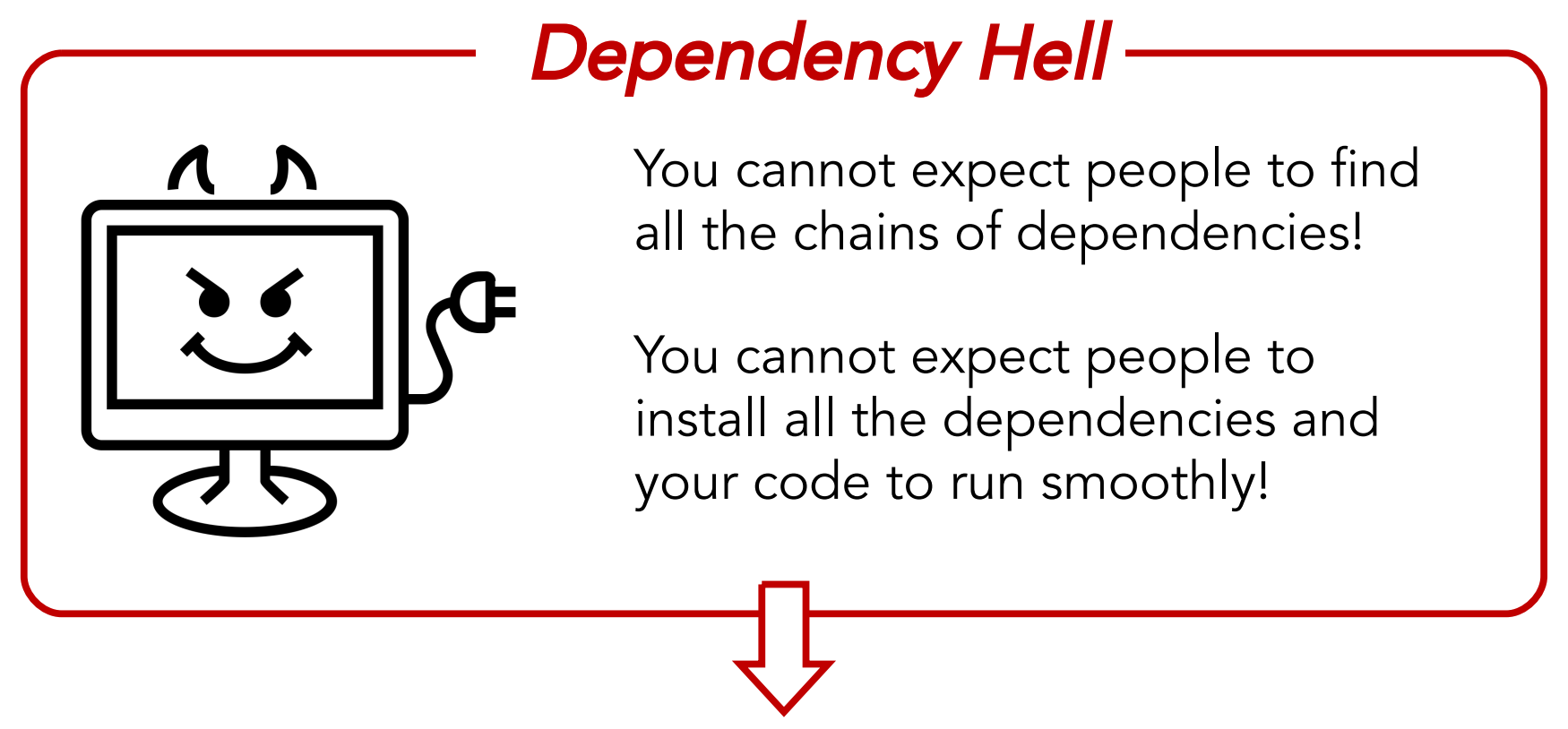

Gap: tools that can automatically capture all the dependencies in the original environment and automatically set them up in another environment 


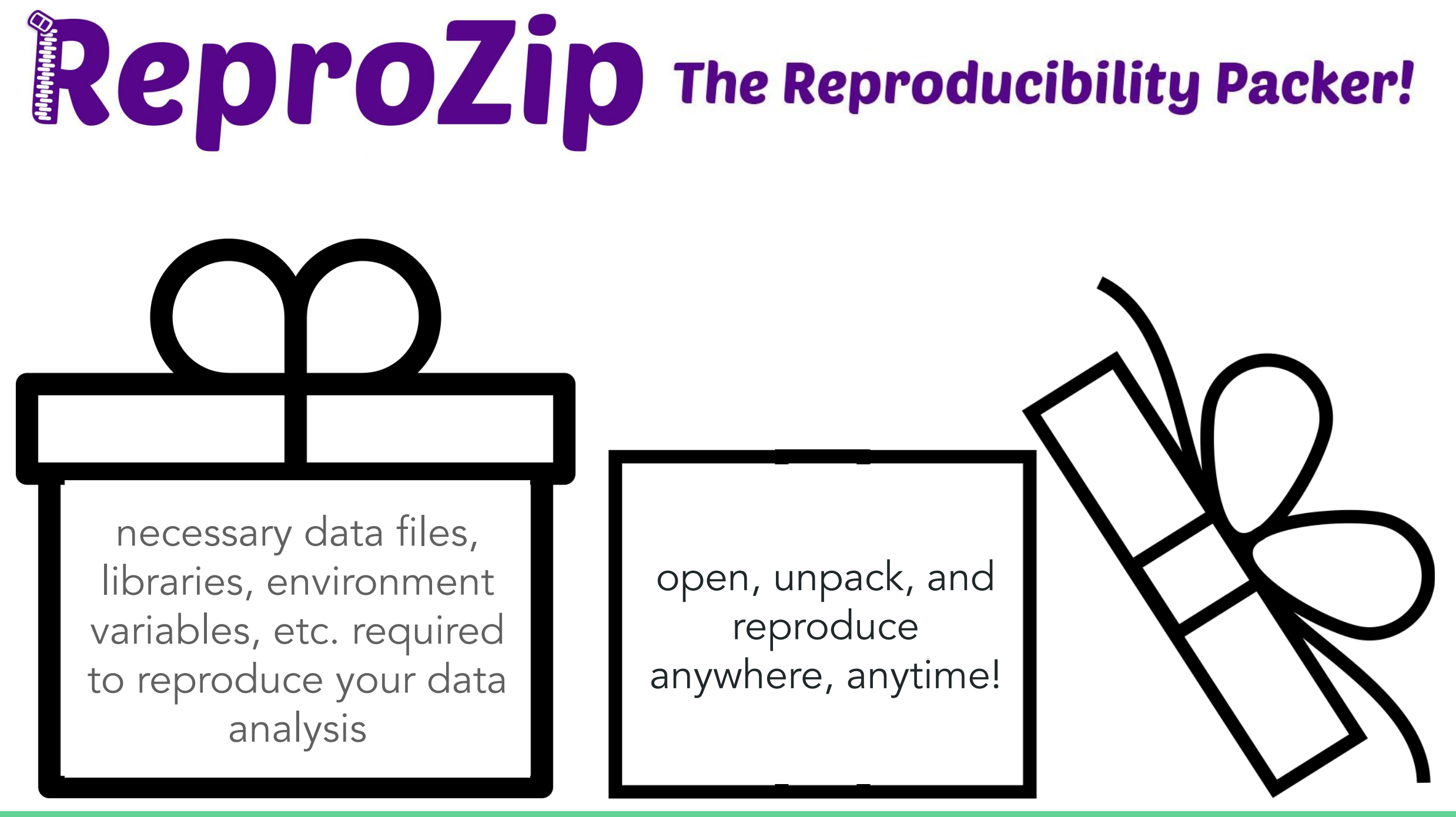




\section{Packing Your Work}

Computational Environment $\boldsymbol{E}$ (Linux)

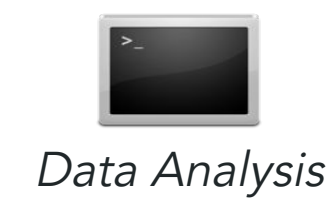

(e.g. Python, R)
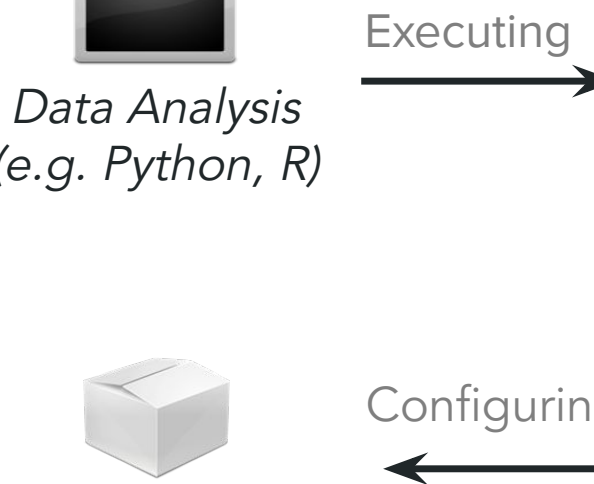

Data Analysis

Package

(.rpz file)
Configuring

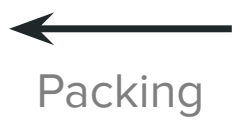

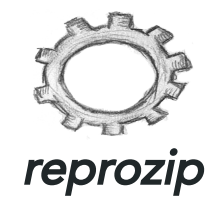

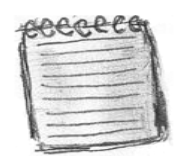

Configuration

File

\section{Experiment Provenance}

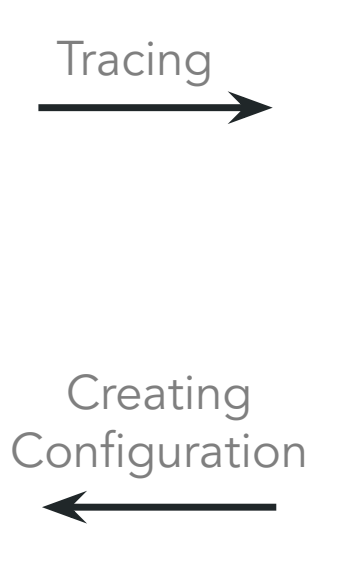

\section{Data}

Input files, output files, parameters Workflow

Executable programs and steps

Environment

Environment variables, dependencies, ... 


\section{ReproZip can pack:}

Data analysis scripts / software (any language, you name it!)

Graphical tools

Interactive tools

Client-server applications (including databases)

Jupyter notebooks

MPI experiments (setting up the experiment can be involved but...)

and much more!

\section{Current Use Cases:}

\section{Academic Use Cases}

- Recommended by the Information Systems Journal, Reproducibility Section

- Recommended by the ACM SIGMOD Reproducibility Review

- Listed on the ACM Artifact Evaluation Process Guidelines

Outside Project Integration

- Integrated as a component of CoRR

- Archiving data journalism apps, e.g.: Stacked Up

- Used by neurodocker to build minimal Docker images

and many more! 


\section{Unpacking Research}

Potentially in a different environment / Operating System

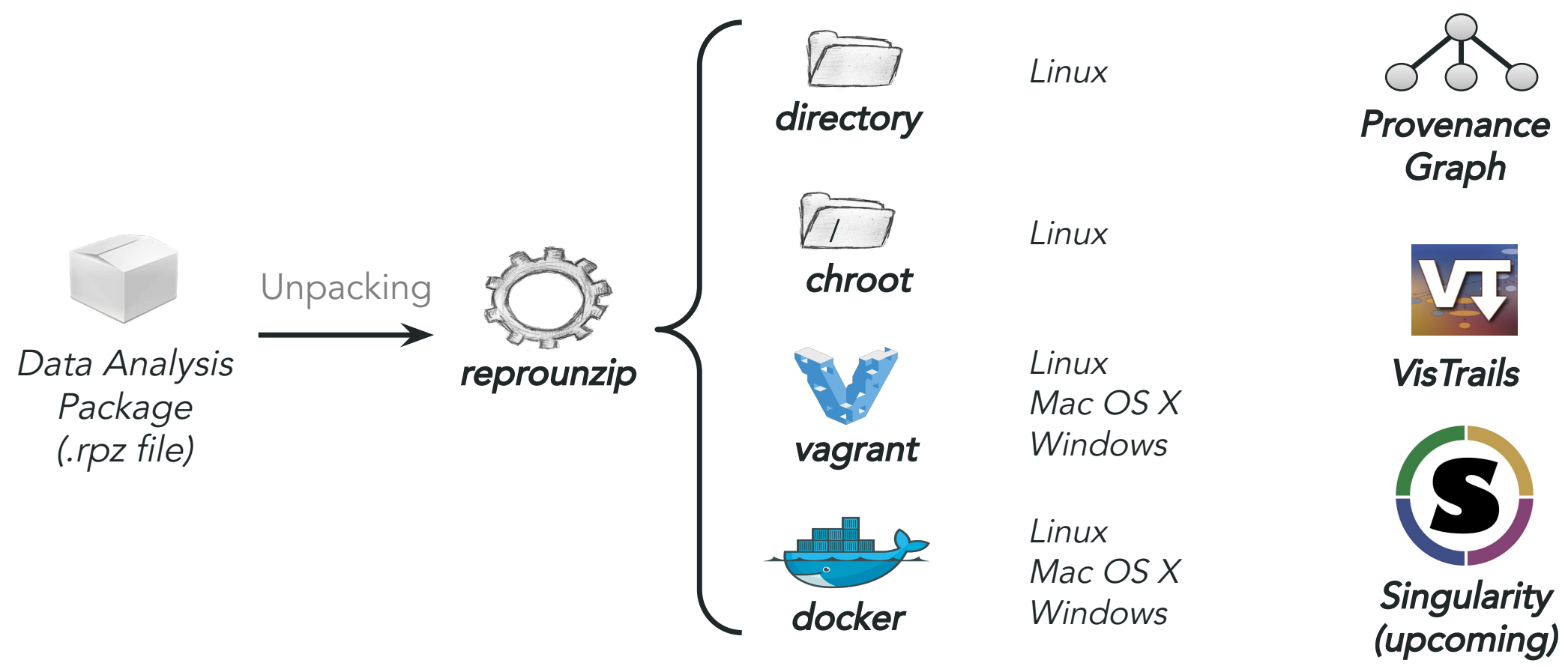




\section{Users can reproduce and extend the original work}

\section{Download Outputs}

\section{OO}

reprounzlp-qt

\begin{tabular}{|c|c|c|}
\hline [0] argo & name: & argo \\
\hline $\begin{array}{l}\text { [1] arg1 } \\
\text { [1] photo.jpg }\end{array}$ & & jenerateClassifier.py \\
\hline $\begin{array}{l}\text { [O] output.jpg } \\
\text { fil mnist-original_mat }\end{array}$ & & (originat) \\
\hline [IO] digits_cls.pkl & 丸 Uplo & ad a replacement $\downarrow$ \\
\hline & & ownload to disk \\
\hline & & Poesed file \\
\hline
\end{tabular}

Upload New Inputs

\begin{tabular}{|c|c|}
\hline \multicolumn{2}{|c|}{ reprounzip-qt } \\
\hline \multirow{6}{*}{$\begin{array}{l}\text { [1] arg0 } \\
\text { [1] arg1 } \\
\text { [i] photo.jpg } \\
\text { [O] outputj.pg } \\
\text { [1] mnist-original.mat } \\
\text { [io] digits_cls.pkl }\end{array}$} & name: output.jpg \\
\hline & Path: ognition/output.jpg \\
\hline & Current: (not created) \\
\hline & Upload a replacement \\
\hline & $\star$ Download to disk $\star$ \\
\hline & Fesest file \\
\hline
\end{tabular}




\section{Why we think our approach is good for preservation}

Well-bundled:

- Captures *everything* your work touches, which is what it needs to rerun! With lots of *extremely* technical metadata!

Generalizable:

- The RPZ format is simple but effective and very generalizable. It can interoperate, be read/accessed by, and run with a lot of software

Future-proofing:

- We can always add/remove unpackers to give users in the future full access to the bundle. As long as there are VMs, containers, or Linux, we can re-execute the bundle contents 


\section{BUT WAIT!}

What if you don't want to download ReproUnzip and Docker or Vagrant?? 
ReproServer to the rescue! No preservation w/o access!

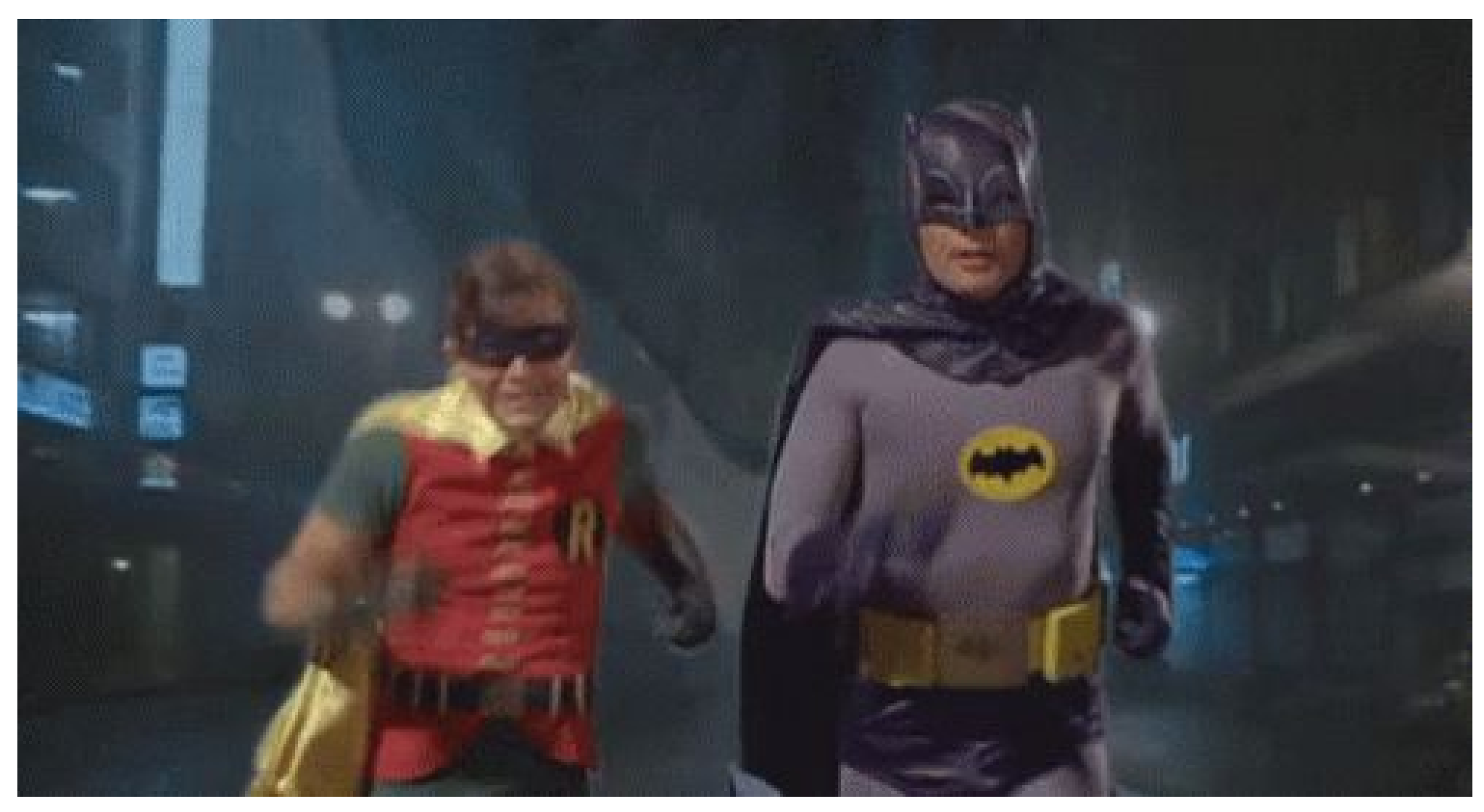


ReproServer, reproducibility in-browser!
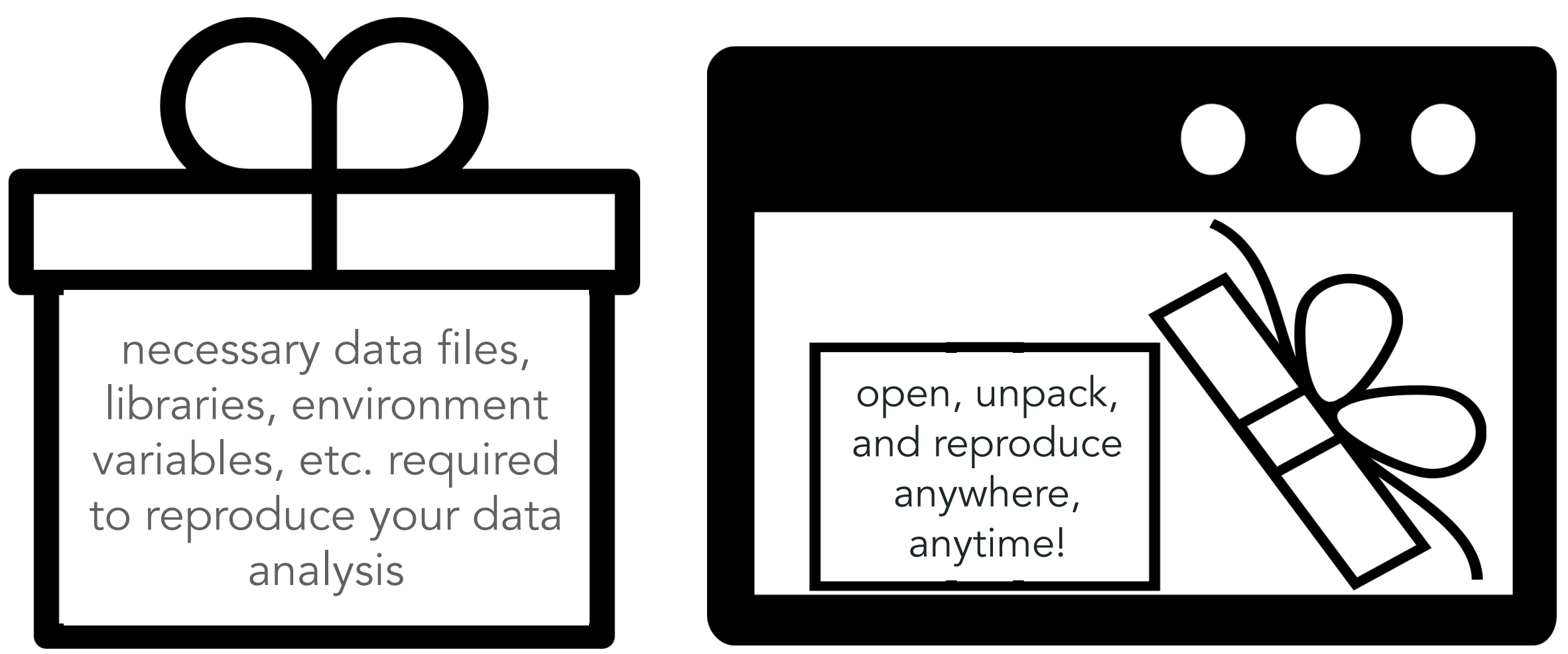


\section{ReproZip + ReproServer $=$ Access to Reproducible Work!}

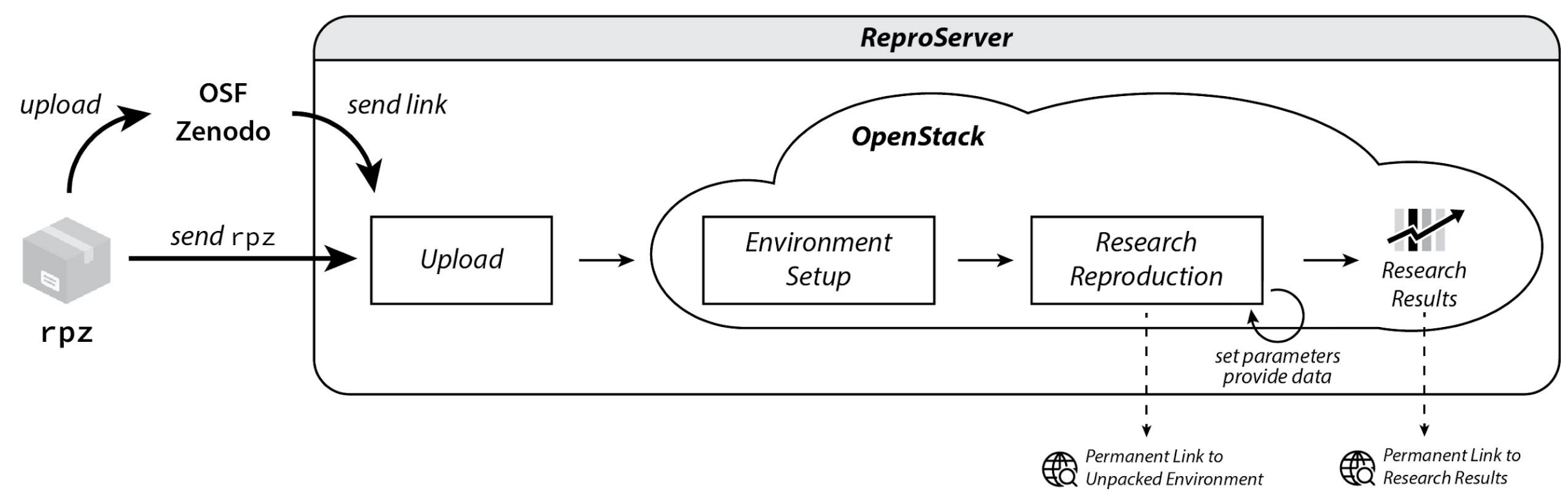




\section{ReproServer -- simplifying access}

- Runs ReproZip packages in the browser, no local software needed

- Allows changing input data \& configs

- Gives you a URL to include in papers to reproduce your experiment

- Offloads archiving responsibility to people who are good at it (ayo)

- No lock-in: build on your laptop, pack automatically, reproduce anywhere

\section{ReproServer Unpack}

\section{Select a package to unpack}

Upload a file

Choose File No file chosen

or provide a package's URL

http://experiment.rpz

Unpack 


\section{Unpacking} local RPZ with $\mathrm{ML}$ Scripts from ReproServer
Select a package to unpack

Unious a fire

cous its Na hile chases

ar eruvlde a packass biat.

\section{Urake}

\section{How to Use This Site}

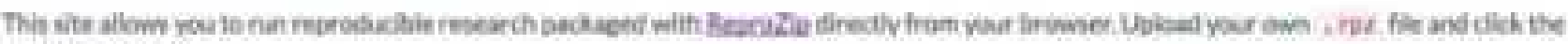
"Uiveco" butsant

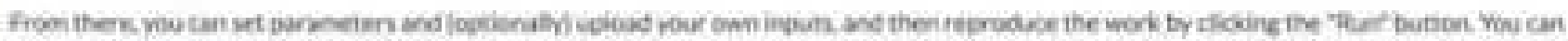

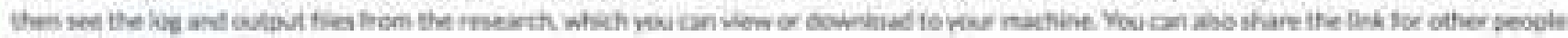
to reprodus your restanti 
Unpacking $R$ plots in RPZ bundle DIRECT

\section{From the OSF}

Select a package to unpack

\section{Uploat a file}

thomatie Nofilechosers

or provide a pachagers URL.

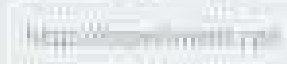

\section{Unouck}

\section{How to Use This Site}

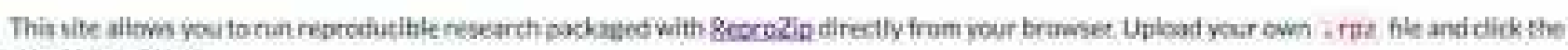
"Unpick" button!

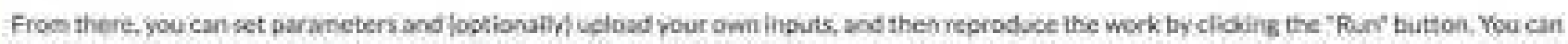
then see the log and outout fies from the research which you can vew or downilost to your mactine You tan also share the firk for other people Wutring 


\section{ReproZip + ReproServer $=$ Preservation + Access!}

- ReproZip provides local, non-locked-in, reproducible packing of research -- easily integrated into existing workflows :)

- ReproServer provides a way for other users to interact with RPZ bundles from the comfort of their browser; easing access, review, and reuse of research materials

- BONUS: see the work in the original computational environment

- BONUS: can read in RPZ bundles from wherever they live (no duplicate upload necessary if in a repository)

- BONUS: can be run locally at your institution (e.g. don't have to rely on centralized infrastructure not controlled by y'all) 


\section{The full ReproZip-ReproServer Ecosystem}

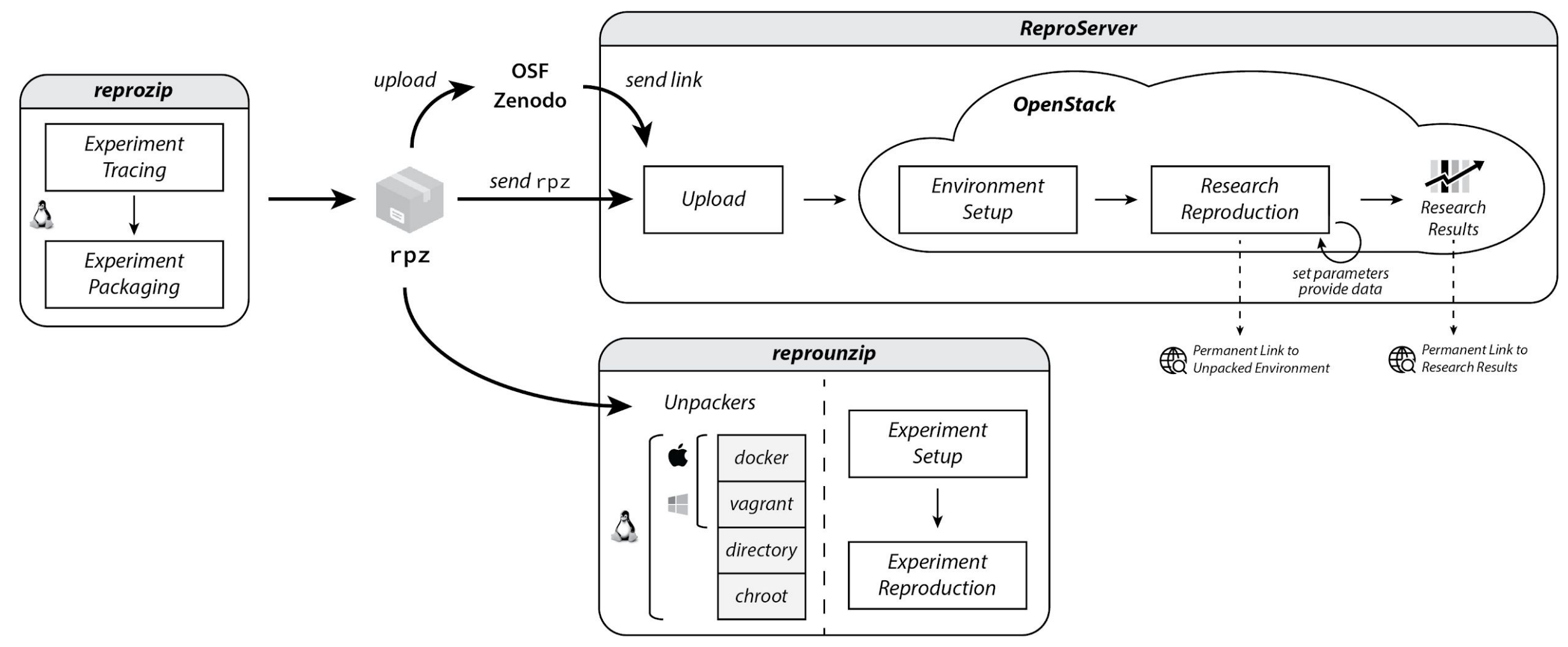




\section{Other Resources for ReproZip \& ReproServer}

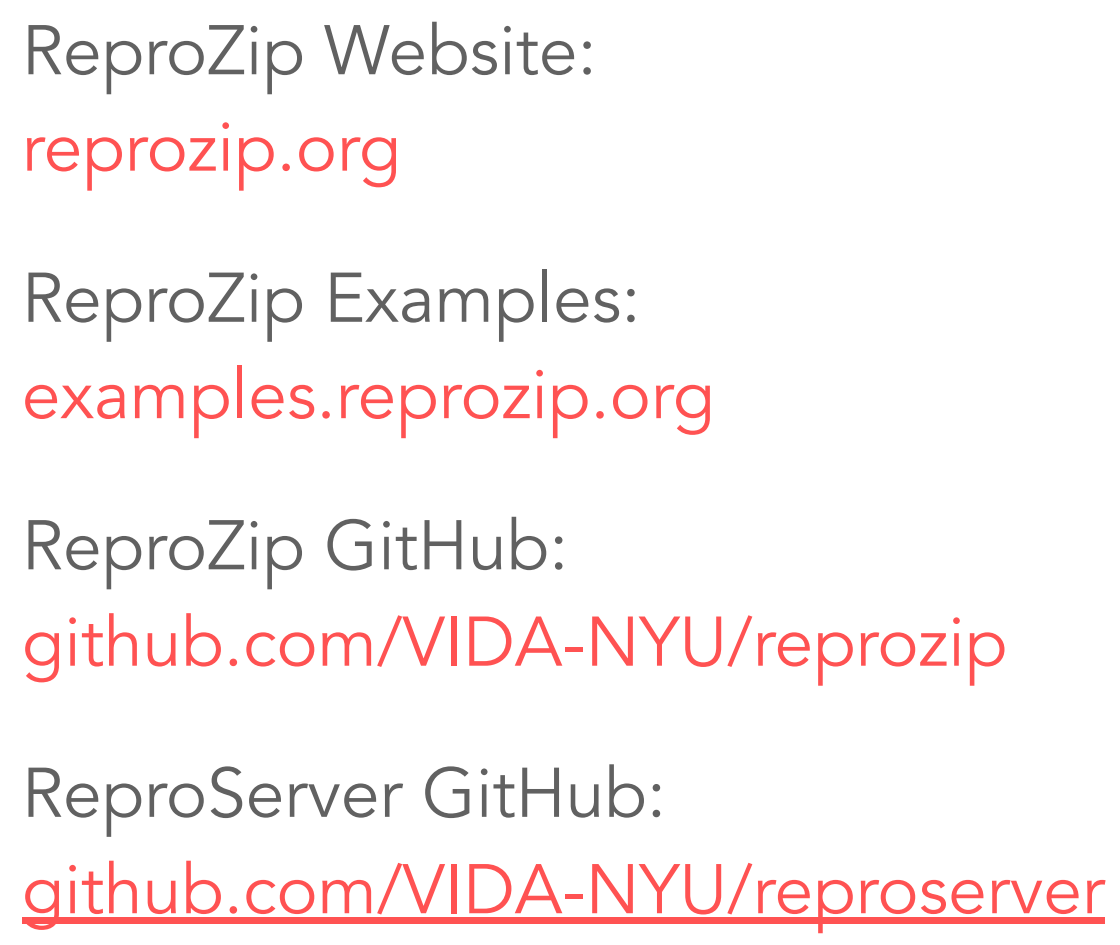

ReproZip packing/unpacking: goo.gl/o1Hqrx

Website packing: goo.gl/yMEOZJ Jupyter notebook packing/replay: goo.gl/NvMHnw

ReproServer demo: goo.gl/Wk7Xnz

ReproServer OSF integration: goo.gl/XfF78z 


\section{Summary:}

- ReproZip provides the preservation + reproducible bundle of work from researchers

- ReproServer provides easier access to the materials of ReproZip bundles in-browser

- No preservation w/o access!

\section{Thank You:}

Fernando Chirigati, ReproZip OG dev \& team member!

Juliana Freire, ReproZip PI

Moore \& Sloan, for the green

Our users, for their feedback and continued help in dev! 